\title{
Integrated Circuit Implementation of a Cortical Neuron
}

\author{
Jayawan H. B. Wijekoon and Piotr Dudek \\ School of Electrical and Electronic Engineering, The University of Manchester, United Kingdom. \\ e-mail: jayawan@iieee.org, p.dudek@manchester.ac.uk.
}

\begin{abstract}
This paper presents an analogue integrated circuit implementation of a cortical neuron model. The VLSI chip prototype has been implemented in a $0.35 \mu \mathrm{m}$ CMOS technology. The single neuron cell has a compact layout and very low energy consumption, in the range of $9 \mathrm{pJ}$ per spike. Experimental results demonstrate the capability of the circuit to generate a realistic spike shape and a variety of spiking and bursting firing patterns. The models of various cortical neuron types are obtained in a single circuit, through the adjustment of two biasing voltages, making the circuit suitable for applications in reconfigurable neuromorphic devices that implement biologically plausible spiking neural networks.
\end{abstract}

\section{INTRODUCTION}

Recently, there has been an increasing research interest in developing computing architectures inspired by biology, in particular based on the models of the animal nervous system [1-7]. In addition to high-level features such as ability to perform complex pattern recognition and motor control, autonomous learning, adaptability etc. also the low-level features, such as robustness against noise and fault tolerance, make the brain-inspired system an attractive alternative computing model which could be appropriate for designing systems in contemporary and future integrated circuit technologies.

The study of the brain reveals that cortical neurons are diverse in their behaviour and many neuron types have been identified, based on electrophysiological characterisations [811]. Hence, in addition to the structural complexity of the network, the heterogeneity of neuronal responses and their complex non-linear oscillatory nature has made it a difficult and challenging task to build large-scale massively parallel VLSI networks that closely resemble the circuits of the cortex. While it remains an open question, at which level of abstraction neural circuits should be modeled to yield a practical computational architecture, the implementation of basic cells, capturing temporal dynamics exhibited by spiking neurons, has been a subject of on-going research interest [1219]. Table I summarizes properties of various implementations found in the literature. Silicon area needed to implement the circuitry is an important consideration for VLSI design, and while the direct comparisons are difficult without considering implementation details, we have included transistor count as an indication of the overall circuit area requirements. The Integrate and Fire (I\&F) neuron cells typically use approximately 20 transistors to implement an adaptive neuron [12] [13]. More realistic circuits, implementing conductancebased neuron models have been reported in the literature [14] [15], however, these circuits use a larger number of transistors. Several other implementations have been proposed [16-20] that are based on mathematical models that capture some of the features of the neuron's oscillatory behaviour. In [21] we have proposed a novel circuit model, which allows the implementation of a cortical neuron using only 14 transistors. The spiking shape produced by the circuit is biologically plausible, and a variety of spiking patterns such as regular spiking, fast spiking, low threshold spiking, intrinsic bursting, chattering etc. can be obtained. In this paper, we report the results obtained from the integrated circuit implementation of the proposed neuron circuit. The circuit is briefly introduced, the chip design is overviewed, and experimental results are presented and discussed.

TABLE I. REVIEW OF VLSI NEURON MODELS.

\begin{tabular}{|l|c|c|c|c|}
\hline \multicolumn{1}{|c|}{ Neuron model } & $\begin{array}{c}\text { Approximate } \\
\text { Number of } \\
\text { transistors }\end{array}$ & $\begin{array}{c}\text { Spiking } \\
\text { pattern }\end{array}$ & $\begin{array}{c}\text { Biological } \\
\text { plausible } \\
\text { spike shape }\end{array}$ & Reference \\
\hline Conductance-based & $27-30+$ & Simple spike & good & {$[14]$} \\
\hline Integrate-and-fire & $18-20$ & Simple spike & fair & {$[13]$} \\
\hline FitzHugh-Nagumo & 20 & Oscillatory & envelope & {$[16]$} \\
\hline Morris-Lecar & 20 & Oscillatory & envelope & {$[17]$} \\
\hline Resonate-and-Fire & 20 & Oscillatory & pulse & {$[18]$} \\
\hline Hindmarsh-Rose & 90 & Bursting & fair & {$[19]$} \\
\hline Proposed model & $\mathbf{1 4}$ & All types & good & \\
\hline
\end{tabular}

\section{THE CIRCUIT}

The proposed circuit is loosely based on the Izhikevich neuron model [11], which uses two differential equations and a reset mechanism to generate the required oscillatory behaviour. In our model, we have replaced the simple mathematical equations with ones more suitable for implementation using CMOS technology, exploiting the underlying nonlinear characteristics of MOS transistors to implement the neuron using as few transistors as possible. The circuit is presented in Figure 1. The implemented neuron model consists of two state variables: "membrane potential" $(V)$ and "slow variable" $(U)$, that are represented by voltages across capacitors $\mathrm{C}_{\mathrm{v}}$ and $\mathrm{Cu}_{\mathrm{u}}$ respectively. The circuit comprises three functional blocks: membrane potential 
circuit, slow variable circuit and comparator circuit (the transistors M1 and M2 are shared by the membrane potential and slow variable circuits). In the membrane potential circuit, the capacitor $\mathrm{C}_{\mathrm{V}}$ integrates the postsynaptic input current, plus internal currents which depend on the state of the cell. Similarly, in the slow variable circuit the capacitor $\mathrm{C}_{\mathrm{u}}$ integrates the currents that non-linearly depend on $U$ and $V$. The comparator detects the spike and generates pulses $\left(V_{A}\right.$ and $V_{B}$ ) that perform the after-spike reset. Various spiking and bursting firing patterns are obtained by tuning two voltage parameters, $V_{c}$ and $V_{d}$, which control the reset mechanism. A more detailed explanation of the circuit behaviour, circuit analysis using phase plane dynamics and mathematical equations of the model are provided in [22].

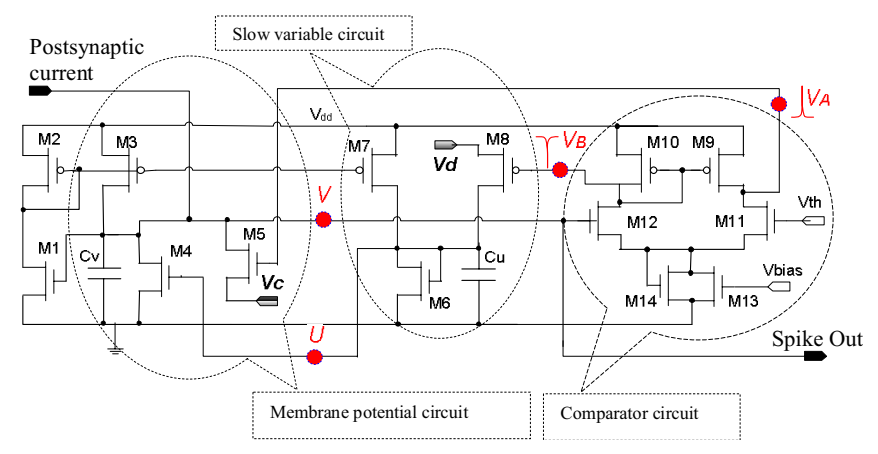

Figure 1. The compact silicon cortical neuron circuit.

\section{INTEGRATED CIRCUIT IMPLEMENTATION}

To experimentally verify the behaviour of our neuron cell we have designed an integrated circuit, fabricated in a $0.35 \mu \mathrm{m}$ CMOS technology. The chip contains 202 neuron cells, with varied circuit parameters (transistor sizes and capacitances) to confirm the simulation results related to transistor scaling and variability [21]. A microphotograph showing the chip layout, as well as individual cells is shown in Figure 2. The purpose of the chip was to experimentally verify the spiking behaviour of a single cell, and thus the cells are individually accessible and do not form any network. In addition to the neuron cells the chip contains required multiplexers, buffers and simple synaptic circuitry to generate excitatory and inhibitory postsynaptic currents. The different neurons are provided with three different types of output buffers to feed the membrane potential signal to the output pads, These types include single stage buffering with two nMOSFETs, double stage buffering with two nMOSFETs and operational amplifier buffering. The circuit also contains a multiplexing unit that selects one neuron at a time. Some cells are designed with an additional external membrane potential resetting circuit using a single transistor. All the experimental results presented in the following sections are taken from a single neuron cell, and its circuit parameters are: $(\mathrm{W} / \mathrm{L})_{\mathrm{Ml}}=(2.3 / 1),(\mathrm{W} / \mathrm{L})_{\mathrm{M} 2}=(2.3 / 1)$, $(\mathrm{W} / \mathrm{L})_{\mathrm{M} 3}=(2.3 / 1), \quad(\mathrm{W} / \mathrm{L})_{\mathrm{M} 4}=(1.3 / 22), \quad(\mathrm{W} / \mathrm{L})_{\mathrm{M} 5}=(5.3 / 1)$, $(\mathrm{W} / \mathrm{L})_{\mathrm{M} 6}=(1.3 / 18), V_{t h}=1.70 \mathrm{~V},(\mathrm{~W} / \mathrm{L})_{\mathrm{M} 7}=(1.3 / 14),(\mathrm{W} / \mathrm{L})_{\mathrm{M} 8}=$ $(1.3 / 1), V_{d d}=3.3 \mathrm{~V}, V_{\text {bias }}=0.6 \mathrm{~V}, C_{v}=0.1 \mathrm{pF}, C_{u}=1 \mathrm{pF}$.

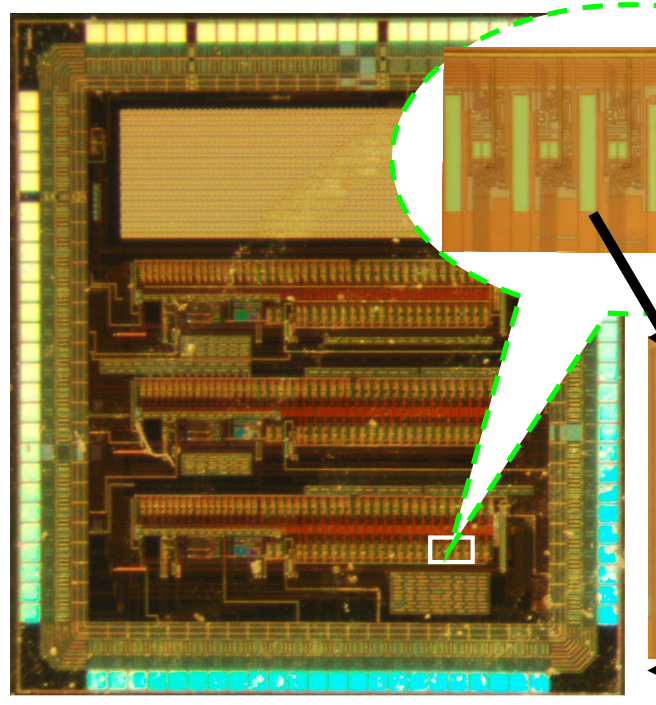

(a)

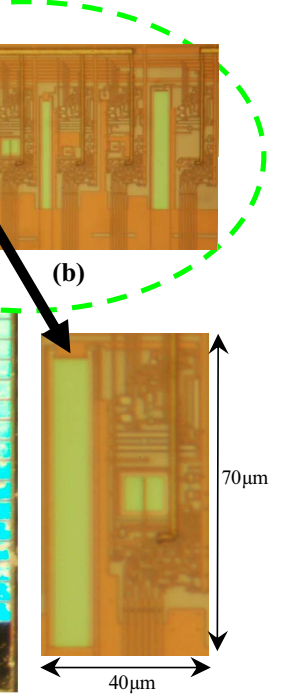

(c).
Figure 2. Photograph of the fabricated device: (a) chip with 202 neurons having different circuit parameters; (b) six different neuron cells; (c) a single neuron including an output buffer and control circuit.

\section{EXPERIMENTAL RESULTS}

The synaptic input was applied to the circuit using a pulse generator, and the biasing parameters were set using programmable voltage supplies. The membrane potential waveforms (buffered using on-chip operational amplifiers) were recorded using a digitising oscilloscope. Figure 3 shows different responses of the circuit to a postsynaptic input current step of $0.1 \mu \mathrm{A}$ and their respective parameters of the tuning voltages $V_{c}$ and $V_{d}$ are provided in Figure 4. The circuit mimics various types of cortical neuron firing patterns: chattering, (CH), intrinsic bursting (IB), low-threshold spiking (LTS), fast spiking (FS) and regular spiking (RS). Brief definitions of each of these firing patterns are presented in [22]. It should be noted that the circuit does not operate in biological real-time, but on approximately $10^{4}$ faster time scale. For comparison purpose, scaled time domain is considered in order to adopt biological classifications methods given in [8]. The classification of spiking and bursting firing patterns using inter-spike interval histograms obtained from the simulations was presented in [21], frequency of spiking and the accommodation (spike frequency adaptation index) were used to further classify these neurons.

The adaptation index measures the accommodation of the firing pattern, i.e. the progressive decrease in firing frequency despite the maintained depolarization. The adaptation index is calculated as $100 \times\left(1-F_{a d} / F_{1}\right)$, where $F_{1}$ corresponds to the firing rate of the first inter-spike interval and $F_{a d}$ is the adapted firing rate [8]. The approximate values of delay $(\delta t)$ between the start of the supra-threshold current injection and the first spike of the spike train, adaptation index and frequency of spiking values for each of RS, LTS and FS type firing patterns are provided in Table II. 


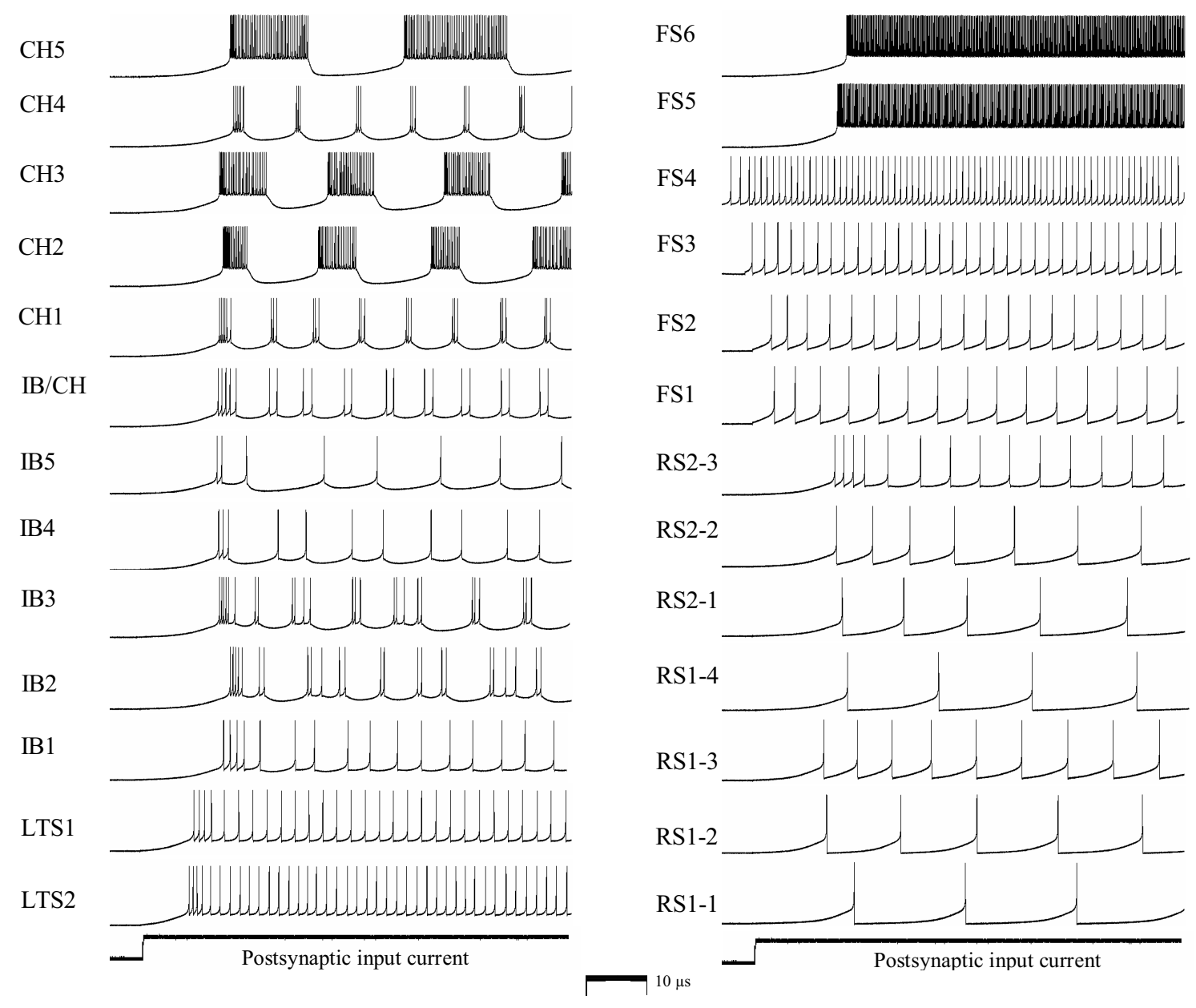

Figure 3. Experimental waveforms of CH, IB, FS, LTS RS1 and RS2 cells. Each plot shows voltage response of the fabricated circuit to a $0.1 \mu \mathrm{A}$ of step current. Parameters $V c$ and $V d$ of each response are provided in Figure 4.

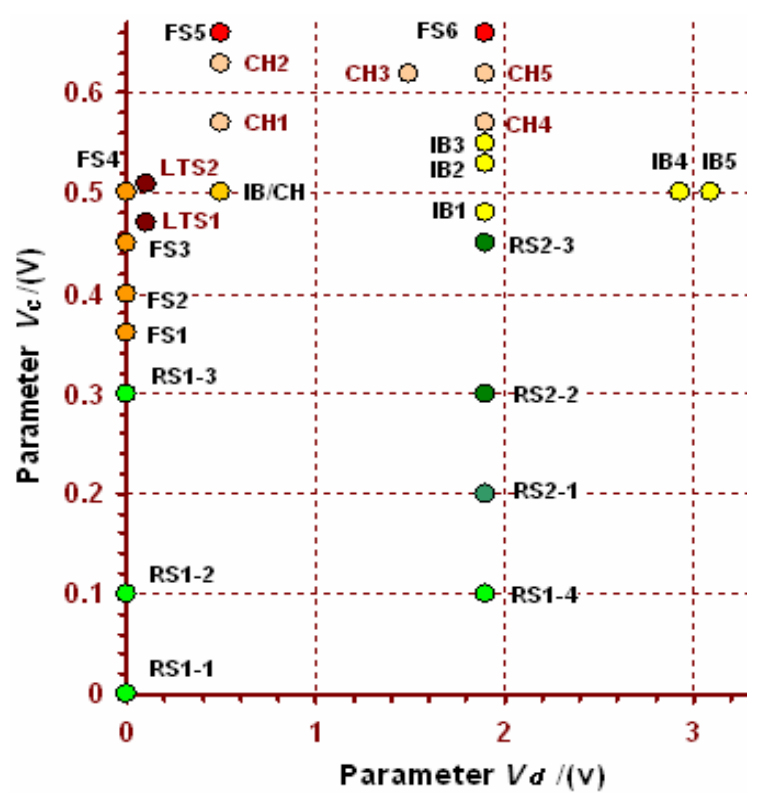

Figure 4. Parameters $V c$ and $V d$ that were used to obtain the cortical neuron firing patterns given in Figure 3.
TABLE II. NEURAL PROPERTIES OF RS, LTS AND FS FIRING PATTERNS PROVIDED IN FIGURE. 3.

\begin{tabular}{|l|c|c|c|l|}
\hline Label & $\begin{array}{c}\text { Delay }^{*} \\
\mathbf{\delta t} \\
(\approx \mathbf{i n} \boldsymbol{\mu s})\end{array}$ & $\begin{array}{c}\text { Adaptation } \\
\text { Index } \\
(\approx \text { in } \%)\end{array}$ & $\begin{array}{c}\text { Frequency of } \\
\text { spiking } \\
(\approx \text { in kHz })\end{array}$ & Type \\
\hline RS1-1 & 17 & 0 & 50 & RS-1 \\
\hline RS1-2 & 13 & 13 & 70 & RS-1 \\
\hline RS1-3 & 12 & 24 & 130 & RS-1 \\
\hline RS1-4 & 16 & 22 & 51 & RS-1 \\
\hline RS2-1 & 15 & 35 & 65 & RS-2 \\
\hline RS2-2 & 14 & 44 & 90 & RS-2 \\
\hline RS2-3 & 14 & 72 & 190 & RS2 \\
\hline LTS1 & 9 & 72 & 300 & LTS \\
\hline LTS2 & 8 & 65 & 480 & LTS \\
\hline FS1 & 4 & 21 & 220 & FS \\
\hline FS2 & 3 & 22 & 280 & FS \\
\hline FS3 & 1 & 4 & 400 & FS \\
\hline FS4 & $<1$ & 2 & 1000 & - \\
\hline FS5 & 14 & 22 & 5500 & FS \\
\hline FS6 & 16 & 35 & 6300 & FS \\
\hline
\end{tabular}

*Delay between the start of the supra-threshold stimuli and the initial spike 
It can be seen that all the firing patterns across $V_{d}=0 \mathrm{~V}$ are weak-accommodating resulting in either RS1 [9] or FS type firing patterns. The FS4 type neuron continues its spiking even after the supra-threshold current is removed, however, it shuts if the inhibitory postsynaptic current is provided. The FS1, FS2, FS3 and the rest of the firing patterns where $V_{d}=0$ and $0.36 \mathrm{~V}<V_{c}<0.5 \mathrm{~V}$ behave as a FS type and their frequency of spiking lies in between $200 \mathrm{kHz}$ to $800 \mathrm{kHz}$. In Figure 4, the approximate parameter space area where $0.2 \mathrm{~V}<V_{d}<3.25 \mathrm{~V}$ and $V_{c}<0.4 \mathrm{~V}$ results in the RS neuron type and when $V_{c}$ increases from 0 to $0.4 \mathrm{~V}$ the frequency of spiking and adaptation index values increase. The parameter space area where $0.2 \mathrm{~V}<V_{d}<3.25 \mathrm{~V}$ and $0.45 \mathrm{~V}<V_{c}<0.56 \mathrm{~V}$ results in IB type and different IB firing patterns can be obtained by varying $V_{d}$ and $V_{c}$ appropriately. Similarly, the area where $0.2 \mathrm{~V}<V_{d}<3.25 \mathrm{~V}$ and $0.56 \mathrm{~V}<V_{c}<0.65 \mathrm{~V}$ produces $\mathrm{CH}$ behaviour and various number of spikes in a burst and interbursting frequencies can be obtained by varying $V_{d}$ and $V_{c}$. In the same $V_{d}$ region when $V_{c}$ is greater than $0.65 \mathrm{~V}$ the cell produces a delayed FS firing pattern with higher firing frequency. Variations of firing patterns of the selected RS, $\mathrm{IB}, \mathrm{CH}$, and $\mathrm{FS}$ cell types with the variation of $V_{c}$ across $V_{d}=1.9 \mathrm{~V}$ illustrate the sensitivity of the firing patterns and their properties to the tuning variable $V_{c}$. It is also important to note that further variations of behaviour can be obtained by changing the $\mathrm{W} / \mathrm{L}$ of transistors M4, M6 and M7 [21].

Depending on the type of the firing pattern, the power consumption of a cell can vary between $0.1-60 \mu \mathrm{W}$ and is approximately proportional to the average spiking frequency. The energy per spike provides a figure of merit that allows a fair comparison of power consumption with respect to the circuit's computational performance. In our circuit the energy consumption per spike is $8.5-9.0 \mathrm{pJ}$ (value obtained via postlayout simulations). For comparison, the I\&F circuit described in [13] consumes 3-15 nJ/spike. The high energy efficiency of our implementation is a result of the higher operating frequency, biasing with low dc currents, and the circuit topology that minimizes the current paths that do not directly contribute to the implementation of the circuit dynamics (i.e. charging and discharging of $\mathrm{C}_{\mathrm{u}}$ and $\mathrm{C}_{V}$ [22]). However, it has to be remembered that a complete neural system will need to include synapse models and spike communication mechanisms, which are likely to dominate the energy requirements. It can be also noted, for comparisons, that a simulation of the Izhikevich neuron [11] on a conventional digital hardware platform consumes somewhere in the range of $1 \mu \mathrm{J}$ per spike.

\section{CONCLUSION}

We have presented an integrated circuit implementation of a compact 14-transistor CMOS cortical neuron circuit. It has been verified experimentally that the circuit is capable of generating many types of cortical neuron behaviour, with diversity similar to that of biological neuron cells. The circuit provides a simple, compact and easily configurable universal cortical neuron, with potential applications in the development of massively parallel analogue VLSI neuromorphic chips that closely resemble the circuits of the neocortex.

\section{ACKNOWLEDGEMENT}

This work has being supported by the COLAMN project, EPSRC grant number EP/C010841.

\section{REFERENCES}

[1] Giacomo Indiveri, Elisabetta Chicca, and Rodney Douglas (2006). A VLSI array of low-power spiking neurons and bistable synapses With Spike-Timing Dependent Plasticity. IEEE Trans. on Neural Netw., vol. 17, no. 1, pp. 211-221.

[2] R. J. Vogelstein, U. Mallik, and G. Cauwenberghs (2004). Silicon spike-based synaptic array and address-event transceiver. in Proc. IEEE Int. Symp. Circuits and Systems, pp. 385-388.

[3] J. Schemmel et al. (2007). Modeling Synaptic Plasticity within Networks of Highly Accelerated I\&F Neurons. IEEE International Symposium on Circuits and Systems, pp. 3367-3370.

[4] F. Tenore, R. Etienne-Cummings, and M. Lewis (2004). A programmable array of silicon neurons for the control of legged locomotion. in Proc. IEEE Int. Symp. Circ. and Systems, pp. 349-352.

[5] A. Bofill-i-Petit and A. F. Murray 2004. Synchrony detection and amplification by silicon neurons with STDP synapses. IEEE Trans. Neural Netw., vol. 15, no. 5, pp. 1296-1304.

[6] S. C. Liu and R. Douglas (2004). Temporal coding in a silicon network of integrate-and-fire neurons. IEEE Trans. Neural Netw., vol. 15, no. 5, pp. 1305-1314.

[7] E. Chicca, D. et al. 2003. A VLSI recurrent network of integrate-andfire neurons connected by plastic synapses with long term memory. IEEE Trans. Neural Netw., vol. 14, no. 5, pp. 1297-1307.

[8] L. G. Nowak, R. et al. (2003). Electrophysiological classes of cat primary visual cortical neurons in vivo as revealed by quantitative analyses. J Neurophysiol,vol. 89, pp.1541-1566.

[9] M. Toledo-Rodriguez, et al. (2003). Neocortex: Basic neuron types. in Hand book of brain theory and neural networks, $2^{\text {nd }}$ e., M.A. Arbib (ed), pp719-725, Cambridge, MA:MIT Press.

[10] $\mathrm{H}$ Markram, et al (2004). Interneurons of the neocortical inhibitory system. Nature Reviews Neuroscience, vol. 5, pp 793-807.

[11] E. M. Izhikevich (2003). Simple model of spiking neurons. IEEE Trans. Neural Netw, vol 14, no. 6, pp. 1569-1572.

[12] S. R. Schultz and M. A. Jabri (1995). Analogue VLSI 'integrateandfire' neuron with frequency adaptation. Electronic Letters, vol. 31, no. 16 , pp. $1357-1358$.

[13] G. Indiveri (2003). A low-power adaptive integrate-and-fire neuron circuit.” IEEE Int. Symp. Circuits and Systems, ISCAS, pp IV820-823.

[14] M.Mahowald, R. Douglas (1991). A silicon neuron. Nature, vol. 354, no $6354,19-26$, pp 515-518.

[15] M. F. Simoni and S. P. DeWeerth (1999). Adaptation in a VLSI model of a neuron. IEEE Transactions on circuits and systems-II: Analog and digital signal processing, vol. 46, no. 7, pp 967-970.

[16] B. Linares-Barranco, et.al. (1991). A CMOS implementation of FitzHugh-Nagumo neuron model. IEEE Journ. Solid-St. Circ., vol. 26, no. 7, pp 956-965.

[17] G.N. Patel, S.P. DeWeerth (1997). Analogue VLSl Morris-Lecar neuron" Electronics Letters, vol. 33, n.12, pp 997-998.

[18] K. Nakada, T. Asai and H. Hayashi (2005). A silicon Resonate-and-fire neuron based on the volterra system. Int. Symp. on Nonlinear Theory and its Applications, pp 82-85.

[19] Young Jun Lee et.al (2004). Low power real time electronic neuron VLSI design using subthreshold technique. IEEE Int. Symp. Circuits and Systems, vol. 4, pp IV-744-747.

[20] K. Nakada, T. Asai, and Y. Amemiya (2004). Analog CMOS implementation of a bursting oscillator with depressing synapse. in Proc. ISSNIP '04, pp 503-506.

[21] Jayawan H. B. Wijekoon, Piotr Dudek (2006). Simple Analogue VLSI Circuit of a Cortical Neuron, IEEE Int. Conf. on Electronics, Circuits and Systems, pp.1344-47.

[22] Jayawan H. B. Wijekoon, Piotr Dudek (2007). Spiking and Bursting Firing Patterns of a Compact VLSI Cortical Neuron Circuit, Int. Joint Conf. on Neural Network. pp.1332-7. 